\title{
The superharmonic instability of finite-amplitude water waves
}

\author{
By P. G. SAFFMAN \\ Applied Mathematics, California Institute of Technology, Pasadena, CA 91125
}

(Received 7 January 1985)

Zakharov's (1968) Hamiltonian formulation of water waves is used to prove analytically Tanaka's (1983) numerical result that superharmonic disturbances to periodic waves of permanent form exchange stability when the wave energy is an extremum as a function of wave height. Tanaka's (1985) explanation for the non-appearance of superharmonic bifurcation is also derived, and the non-existence of stability exchange when the wave speed is an extremum is explained.

\section{Introduction}

We consider the stability of finite-amplitude two-dimensional water waves of permanent form to two-dimensional disturbances of the same wavelength, which are called superharmonic. This problem was considered first by Longuet-Higgins (1978a), who showed that gravity waves are stable to such disturbances for $h / \lambda<0.1337$, which was the greatest height for which the calculations were accurate. Extrapolation of the results indicated an exchange of stability for $h / \lambda=0.1388$, which is the height for which the wave speed $c$ first attains a stationary value (in this case a maximum) as a function of wave height. This is expected, since there is a disturbance stationary relative to the wave when $c$ is an extremum. Here $h$ denotes wave height defined as vertical distance between crests and troughs, and $\lambda$ is the wavelength (i.e. horizontal distance between neighbouring crests). An exchange of stability means that in a frame moving with the wave the eigenvalue giving the growth rate of infinitesimal disturbances goes through zero from pure imaginary to real and that the critical eigenvector describes a disturbance that is stationary relative to the propagating wave.

However, Tanaka (1983) reexamined the superharmonic instability of gravity waves and found the surprising result that exchange of stability occurs for a wave height less than that for which $c$ is first stationary. It is, in fact, within numerical error, the wave height for which the energy of the wave, kinetic plus potential, has its first maximum as a function of wave height. Tanaka's result was also unexpected, because the appearance of a zero eigenvalue usually implies, according to simple bifurcation theory, either the existence of a limit point or superharmonic bifurcation to a new branch of solutions with the same wavelength. But the wave of maximum energy is not a limit point (the precise meaning of limit point in the water-wave context will be given below; see the discussion following (3)), and on the other hand superharmonic bifurcation is inconsistent with Garabedian's (1965) theorem that gravity waves are unique if all crests and all troughs are of the same height, and the calculations by Chen \& Saffman (1980), who searched for superharmonic bifurcation into both symmetrical and asymmetrical waves by numerical means and were unable 
to find it. Furthermore, there was no sign of a stability exchange for the wave of maximum speed.

In recent work, Tanaka (1985) has convincingly confirmed his calculations and explained why his finding of an exchange of stability away from a limit point does not contradict the non-existence of superharmonic bifurcation. Briefly, it is due to the algebraic multiplicity and geometrical multiplicity being different and the sole eigenvector that gives the new branch coinciding with the eigenvector for the trivial bifurcation corresponding to horizontal translation. Longuet-Higgins (1984) has shown that the existence of a zero eigenvalue implies for gravity waves that either the wave is at a limit point or the corresponding eigenvector is that for a horizontal shift.

In this note we shall give an analytic proof of Tanaka's results and confirm his prediction that there will be an exchange of stability at every stationary value of the energy, and moreover that these exchanges of stability do not produce superharmonic bifurcation into a physically new type of wave. It will also be shown that exchange of stability does not in general occur when the wave speed $c$ is an extremum.

It should be noted that the distinction between exchange of stability and occurrence of superharmonic instability is real as non-stationary modes may in principle coalesce to give propagating instabilities, although no evidence has yet been found that this occurs for two-dimensional superharmonic disturbances. $\dagger$ It does occur for subharmonic (Longuet-Higgins 1978b) and three-dimensional (McLean et al. 1981) disturbances.

\section{Steady waves}

We employ the Hamiltonian formulation of water waves presented by Zakharov (1968) and apply it to a wave propagating in the $x$-direction with wavelength $2 \pi$. The canonical variables are complex quantities $a_{n}$ and $a_{n}^{*}(-\infty<n<\infty)$, which are linear combinations of the complex Fourier components of the wave elevation $\eta(x, t)$ and velocity potential evaluated on the surface $\psi(x, t)$. The total energy density (kinetic plus potential per unit horizontal length) is $E\left(a_{n}, a_{n}^{*}\right)$. Zakharov showed that the equations for the evolution of the wave could be written in Hamiltonian form as

$$
\frac{\partial \boldsymbol{a}}{\partial t}=-\mathrm{i} \frac{\partial E}{\partial \boldsymbol{a}^{*}}, \quad \frac{\partial \boldsymbol{a}^{*}}{\partial t}=\mathrm{i} \frac{\partial E}{\partial \boldsymbol{a}},
$$

with $a=\left\{a_{n}\right\}$. Changing to a coordinate system moving with speed $c$, the equations take the form

$$
\frac{\partial \boldsymbol{a}}{\partial t}=-\mathrm{i} \frac{\partial \boldsymbol{E}}{\partial \boldsymbol{a}^{*}}+\mathrm{i} \boldsymbol{c} \boldsymbol{l}_{n} \boldsymbol{a}, \quad \frac{\partial \boldsymbol{a}^{*}}{\partial t}=\mathrm{i} \frac{\partial E}{\partial \boldsymbol{a}}-\mathrm{i} \boldsymbol{c} \boldsymbol{I}_{n} \boldsymbol{a}^{*},
$$

where $I_{n}$ denotes the diagonal matrix with $n$ on the $n$th row and $n$th column.

Equilibrium solutions that describe waves of permanent form are therefore described by solutions of the equations

$$
\frac{\partial E}{\partial A^{*}}-c I_{n} A=0, \quad-\frac{\partial E}{\partial A}+c I_{n} A^{*}=0,
$$

where we denote equilibrium values by $A$ and $A^{*}$. It is convenient in this formulation

$\dagger$ Note added in proof: Such instabilities have been predicted and found recently by MacKay \& Saffman; paper in preparation. 
to think of the wave speed $c$ as the independent variable and the Fourier coefficients (and thus the wave height) as dependent variables. (This approach was also adopted by Saffman (1980) in a study of the general properties of subharmonic bifurcation.) If we normalize gravity so that the wave speed $c=1$ for an infinitesimal wave of period $2 \pi$, then nonlinear waves are obtained by finding solutions with $c>1$. If, as we follow the family of solutions by increasing $c$, we find that $c$ has a maximum (and this is the case for gravity waves on deep water), we have a limit point or turning point, and the solution curve bends back in $(A, c)$-space. This would be recognized by $\mathrm{d} A / \mathrm{d} c$ and $\mathrm{d} A^{*} / \mathrm{d} c$ becoming infinitely large.

Owing to the symmetry of inviscid water waves, the energy $E\left(a, a^{*}\right)$ has certain symmetries. In particular, $E$ is invariant under the change $a \rightarrow a^{*}$ and also $a_{n} \rightarrow a_{-n}$. Further, invariance under horizontal translation implies that, if $A=\left\{A_{n}\right\}$ is a solution of the system (3) for some value of $c$, so is $A^{\xi}=\left\{A_{n} \mathrm{e}^{\mathrm{in} \xi}\right\}$ for arbitrary values of the displacement $\xi$. The solution of (3) is therefore not unique. Mathematically, the Jacobian of the system (3) is singular, i.e. the matrix

$$
J=\left[\begin{array}{rc}
M & N \\
-N^{*} & -M^{*}
\end{array}\right]
$$

where $M=\partial^{2} E / \partial A^{*} \partial A-c l_{n}$ and $N^{*}=\partial^{2} E / \partial A \partial A$, has codimension greater than zero. The null vector associated with the invariance under translation is obtained from evaluating $\mathrm{d} A^{\xi} / \mathrm{d} \xi$ at $\xi=0$, and it follows that the right and left eigenvectors of $J$ are

$$
a=\left(I_{n} A,-I_{n} A^{*}\right), \quad a^{\dagger}=\left(I_{n} A^{*}, I_{n} A\right) .
$$

Now $E$ is real, and a steady solution can be uniquely specified by the condition that $A$ and $A^{*}$ are real, i.e.

$$
A(c)=A^{*}(c)
$$

The matrices $\boldsymbol{M}$ and $\boldsymbol{N}$ are then real and symmetric, and the order of the system (3) is halved. The asterisk on $A, M$ and $N$ is now dropped. For the family of steady solutions, the energy $E$ is an implicit function of wave speed given by $E(c)=E(A(c), A(c))$. The variation of $E$ along the family is found from (3) to be given by

$$
\frac{\mathrm{d} E}{\mathrm{~d} c}=2 \frac{\partial E}{\partial A} \cdot \frac{\mathrm{d} A}{\mathrm{~d} c}=2 c I_{n} A \cdot \frac{\mathrm{d} A}{\mathrm{~d} c} .
$$

\section{Perturbations}

Let us now consider time-dependent perturbations of the same wavelength to the steady solution. We write

$$
\boldsymbol{a}=\boldsymbol{A}+\boldsymbol{\epsilon} \boldsymbol{b}(t), \quad \boldsymbol{a}^{*}=A+\epsilon \boldsymbol{b}^{*}(t),
$$

substitute into (2) and linearize in $\epsilon$. This gives the linear equations

$$
-\mathrm{i} \frac{\mathrm{d} b}{\mathrm{~d} t}=-\boldsymbol{M b}-\boldsymbol{N b}^{*}, \quad-\mathrm{i} \frac{\mathrm{d} b^{*}}{\mathrm{~d} t}=\boldsymbol{M} b^{*}+\boldsymbol{N b},
$$

We require solutions of these equations with $b$ and $b^{*}$ complex conjugates, but in order to determine stability or instability it is sufficient to look for solutions with

$$
b=b^{+} \mathbf{e}^{i \sigma t}, \quad b^{*}=b^{-} e^{i \sigma t},
$$


where $b^{+}$and $b^{-}$are not necessarily complex conjugates. Then the eigenvalue $\sigma$ and eigenvector $\left(b^{+}, b^{-}\right)$are solutions of

$$
\left[\begin{array}{cc}
\sigma+M & M \\
-N & \sigma-M
\end{array}\right]\left[\begin{array}{l}
b^{+} \\
b^{-}
\end{array}\right]=0 .
$$

and appropriate linear combinations of the eigenvectors will give solutions that satisfy the complex conjugate condition. It follows trivially from (11) by writing equations for $b^{+} \pm b^{-}$that these vectors satisfy the eigenvalue problems

$$
\begin{aligned}
& \sigma^{2}\left(b^{+}-b^{-}\right)=(M+N)(M-N)\left(b^{+}-b^{-}\right), \\
& \sigma^{2}\left(b^{+}+b^{-}\right)=(M-N)(M+N)\left(b^{+}+b^{-}\right) .
\end{aligned}
$$

This provides a mathematical proof of the result, which can be deduced from invariance under time reflection, that the eigenvalues come in pairs $( \pm \sigma)$ if real or pure imaginary, and quartets $\left( \pm \sigma, \pm \sigma^{*}\right)$ if complex. Note that the matrix on the right-hand side of (12) or (13) is not symmetric, so the values of $\sigma^{2}$ are not necessarily real. For numerical purposes, using (12) or (13) reduces the order of the system by one-half. The analytical questions involve the dimension of the null space of the matrix $\boldsymbol{J}$, in addition to the algebraic multiplicity of the zero eigenvalue, and it then seems more natural to work with the larger system given by (11).

It follows from (4) that $\sigma=0$ is a root of (11), and it is a consequence of time reversal or (12) and (13) that it is a double root. We now consider the criterion for $\sigma=0$ to be a quadruple root, which will occur when two, in general non-zero, eigenvalues vanish for one wave of the family and there is an exchange of stability.

\section{Exchange of stability}

We suppose we are not at a limit point, and calculate the quantity $\mathrm{d} \boldsymbol{A} / \mathrm{d} c$, which is obtained by differentiation of the equilibrium equation (3) with respect to $c$. We have

$$
(\boldsymbol{M}+\boldsymbol{N}) \frac{\mathrm{d} A}{\mathrm{~d} c}=I_{n} A
$$

This equation implies

$$
J\left[\begin{array}{l}
\mathrm{d} A / \mathrm{d} c \\
\mathrm{~d} A / \mathrm{d} c
\end{array}\right]=\left[\begin{array}{r}
I_{n} A \\
-I_{n} A
\end{array}\right] .
$$

We rewrite this to say that the equation

$$
\boldsymbol{J} \boldsymbol{\beta}=\boldsymbol{a}
$$

has a solution $\beta=(\mathrm{d} A / \mathrm{d} c, \mathrm{~d} A / \mathrm{d} c)$. Since, from (5), Ja $=0$, it follows that $\sigma=0$ is a multiple eigenvalue of $\boldsymbol{J}$ with a one-dimensional null space spanned by $\boldsymbol{a}$. The corresponding left eigenvector is $\boldsymbol{a}^{\dagger}$. These two vectors are orthogonal, i.e. $\boldsymbol{a}^{\dagger} \cdot \boldsymbol{a}=\mathbf{0}$.

Now consider the equations

and

$$
\begin{aligned}
& \boldsymbol{J} \boldsymbol{\gamma}=\boldsymbol{\beta} \\
& \boldsymbol{J} \boldsymbol{\delta}=\boldsymbol{\gamma} .
\end{aligned}
$$

If these equations have solutions, then the eigenvector $a$ has algebraic multiplicity at least four, and exchange of stability occurs. The necessary and sufficient condition for them to have solutions is that the right-hand sides are orthogonal to $a^{\dagger}$. ('These results for matrices are easily proved by considering the Jordan normal forms (see 
e.g. Gantmacher 1960 , for a $4 \times 4$ matrix and examining the various possibilities). Note that, from the symmetry, $\gamma$ can be chosen to have the form $\left(c^{+},-c^{+}\right)$, and hence the right-hand side of (18) is trivially orthogonal to $a^{\dagger}$ when (17) has a solution. It is therefore sufficient to consider just (17).

Now the condition for (17) to have a solution is

$$
I_{n} A \cdot \frac{\mathrm{d} A}{\mathrm{~d} c}=0
$$

But this is exactly from (7) the condition for the energy to have an extremum. Thus when the energy is a maximum or minimum, the eigenvalue problem for the growth rate of infinitesimal perturbations has a quadruple zero (i.e. an algebraic multiplicity of four), but just one independent eigenvector (i.e. a geometrical multiplicity of one), which corresponds to translation. This is exactly the phenomenon found by Tanaka (1985) in his numerical calculations for gravity waves at the first maximum of $E$ and suggested for the subsequent minimum. The present deduction shows that this behaviour is general for any Hamiltonian system with the appropriate symmetries. It would apply, for example, to gravity-capillary waves if the energy has an extremum.

Since the bifurcation that takes place at a zero eigenvalue is such that the tangent vector of the solution branch is a linear combination of the corresponding null-space eigenvectors, there is indeed superharmonic bifurcation associated with the exchange of stability at the extremum values of the energy, but it is a trivial bifurcation corresponding to translation, and not the appearance of new types of waves. Garabedian's theorem is not violated, and Chen \& Saffman's calculations are confirmed.

We have from (12) and (13) that $\sigma^{2}$ is the eigenvalue of a matrix. It follows that $\sigma^{2}$ remains real unless there is further coalescence of eigenvalues, i.e. a multiplicity of six. There is no reason to expect this happens in general, and hence after the exchange of stability $\sigma^{2}$ will be negative and $\sigma$ will be pure imaginary. Thus the unstable modes for larger values of $c$ will be growing waves which move with the same speed as the undisturbed wave.

\section{Behaviour at the limit point}

Let us change the independent variable from $c$ to $h$, where $h$ is the wave height or some equivalent monotonic measure of arclength along the family of steady solutions. The equation (15) describing the change of $\boldsymbol{A}$ along the solution curve is

$$
(\boldsymbol{M}+\boldsymbol{N}) \frac{\mathrm{d} A}{\mathrm{~d} h}=I_{n} A \frac{\mathrm{d} c}{\mathrm{~d} h} .
$$

At a limit point, $\mathrm{d} c / \mathrm{d} h=0$, and hence $(\mathrm{d} A / \mathrm{d} h, \mathrm{~d} A / \mathrm{d} h)$ is a right null vector of $J$. This vector and $a$ are then two independent null eigenvectors, and 0 is a double eigenvalue. However, (16) no longer has a solution, and we cannot infer a quadruple zero and an exchange of stability. $\uparrow$ Thus the behaviour at the limit point is special in that the dimension of the null space is two, but there are no further zeros of the eigenvalue and the double zero corresponding to translation invariance remains a double zero. The extra eigenvector describes the continuation along the regular

If I wish to thank Dr P. A. E. M. Janssen for providing a counter-example to a fallacious argument that a quadruple zero existed at the limit point. 
solution branch. The plausible argument that the existence of a limit point implies an exchange of stability fails in this case because it implicitly assumes that other zero eigenvalues do not exist, which is not so here owing to the Galilean invariance.

A simple example which shows explicitly that a Hamiltonian with the appropriate symmetries has the properties described in this paper will be published elsewhere (Zufiria \& Saffman 1985).

I have benefited from discussions with Dr H. C. Yuen, Dr P. A. E. M. Janssen and Mr J. Zufiria. This work was supported by the Office of Naval Research (N00014-79-C-0412 NR062-639).

\section{REFERENCES}

Chen, B. \& Saffman, P. G. 1980 Numerical evidence for the existence of new types of gravity wave of permanent form on deep water. Stud. Appl. Maths. 62, 1-21.

Gantmacher, F. R. 1960 Matrix Theory. Chelsea.

Garabedian, P. R. 1965 Surface waves of finite depth. J. d'Anal. Math. 14, 161-169.

LONGUET-Higains, M.S. $1978 a$ The instabilities of gravity waves of finite amplitude in deep water. I. Superharmonics. Proc. R. Soc. Lond. A 360, 471-488.

LoNGUET-Higgins, M. S. $1978 b$ The instabilities of gravity waves of finite amplitude in deep water. I. Subharmonics. Proc. $R$. Soc. Lond. A 360, 489-505.

Lonavet-Higgins, M. S. 1984 On the stability of steep gravity waves. Proc. R. Soc. Lond. A 396, 269-280.

Mclean, J. W., Ma, Y. C., Martin, D. U., Saffman, P. G. \& Yuen, H. C. 1981 Three dimensional instability of finite amplitude water waves. Phys. Rev. Lett. 46, 817-820.

SAFrman, P. G. 1980 Long wavelength bifurcation of gravity waves on deep water. J. Fluid Mech. $101,567-581$.

Tanaka, M. 1983 The stability of steep gravity waves. J. Phys. Soc. Japan 52, 2047-3055.

Tanaka, M. 1985 The stability of steep gravity waves. Part 2. J. Fluid Mech. 000, 000-000.

ZAKHAROV, V. E. 1968 Stability of periodic waves of finite amplitude on the surface of a deep fluid. J. Appl. Mech. Tech. Phys. 2, 190-194.

ZuFiria, J. \& Safrman, P. G. 1985 An example of stability exchange in a Hamiltonian wave system. Stud. Appl. Maths (to appear). 